\title{
Calling for help: teaching medical students about situation, background, assessment and recommendation
}

\author{
Authors: Claire Gibbons and Rebecca Darge
}

\section{Aims}

To pilot a new tutorial to improve medical students' confidence in using situation, background, assessment and recommendation (SBAR) for clinical communication.

\section{Methods}

Over 3 months we facilitated high-fidelity simulation sessions for final year medical students. During this we observed that students struggled to give a good-quality handover. Colleagues across two sites reported similar observations when surveyed. We designed a 1-hour tutorial, to be delivered by two faculty members, to teach students to: describe and evaluate SBAR; critically appraise model SBAR procedures; and develop SBAR good practice through lowfidelity simulation.

We evaluated session outcomes using student questionnaires and compared results with answers from students who did not attend.

\section{Results}

We invited 20 final-year medical students on their acute specialty placements to attend the tutorial; 10 attended. Students reported having limited opportunity to practice using SBAR previously. Of those who attended the tutorial, six reported significantly improved confidence in using SBAR, and four reported slightly improved confidence. Qualitative feedback was largely positive. Students liked the use of simulation, receiving individual feedback and smallgroup learning in a positive environment. They felt the session could be improved by more individual practice. Both students who did and did not attend the tutorial felt that their acute specialty clinical placements had improved their confidence in using SBAR. However, students who attended the tutorial reported greater overall confidence in using SBAR, and saw a greater increase in their confidence, than their peers who did not attend the tutorial.

Taking feedback into account, the tutorial has been assimilated into the mandatory teaching timetable with consistently positive feedback. It is also being incorporated into postgraduate teaching.

\section{Conclusion}

Medical students benefit from opportunities to practice communication skills in simulated settings. By recognising and acting upon gaps in student abilities it is possible to introduce new, effective and relevant teaching. It should not be assumed that students will develop key communication skills by clinical exposure alone; these skills require teaching and practice with dedicated feedback. Simulation can provide a safe environment for this to occur and can facilitate experiential learning.

\section{Conflict of interest statement}

None declared. 